\title{
Investigation of triaxial stress state in retained austenite during quenching of a low alloy steel by in situ X-ray diffraction
}

\author{
Jérémy Epp \\ Stiftung Institut fuer Werkstofftechnik, Badgasteiner Str. 3, 28359, Bremen, Germany \\ epp@iwt-bremen.de
}

Keywords: in situ X-ray diffraction, retained austenite, quenching, triaxal stress state, AISI 52100

\begin{abstract}
In situ XRD measurements were performed at ESRF, Grenoble, France (ID11) during quenching of a ball bearing steel AISI 52100 (100Cr6) with varying carbon content in solution. The evolution of austenite lattice parameter during cooling is nearly linear until Ms is reached and then, a divergent behavior can be observed. Assuming that the extrapolation of the linear range to room temperature gives the stress-free lattice spacing, an increasing compressive hydrostatic stress state is resulting. A strong effect of the carbon content was found. These results were confirmed by theoretical calculations based on data from the literature.
\end{abstract}

\section{Introduction}

Martensitic transformation in steels has now been investigated for more than 100 years [1- 3]. The interest in martensitic transformations is still very high as numerous industrial applications use this transformation to improve wear, mechanical and fatigue properties of parts in engineering components [4]. Moreover, new interest on fundamentals of martensitic transformations appeared in the last decades with the development of computer simulation, where kinetics, distortions and other phenomenon has to be well described to reach reliable simulation results $[5,6]$.

Few studies can be found in the literature about residual stresses in retained austenite during or after quenching of steel. Several authors describe the residual stress state within retained austenite existing at room temperature as a hydrostatic residual stress state under high compressive stresses $[7,8,9]$. The reason for this would be the very large volume expansion associated with the martensititic transformation ( $>3$ Vol. \%). Due to shear-processes during the martensitic transformation, local residual stresses will not be purely hydrostatic in a single austenite region. However, X-ray diffraction techniques give average information of thousands (millions) of crystallites which in general are randomly oriented, and therefore the average information contained in the measured area might be predominantly hydrostatic.

In situ X-ray diffraction analysis has become a powerful method of materials characterization stimulated by constant advances in instrumentation and data processing. This method allows, contrarily to dilatometry or resistivity measurements, to obtain time-resolved quantitative information about every single phase present in the investigated material [10].

In the present study, in situ X-ray diffraction experiments were performed at ESRF on Beamline ID11 during heat treatment of $100 \mathrm{Cr} 6$ steel with varying parameters. By variations of the austenitizing temperature, different carbon contents in solution were observed, leading to different behavior during quenching. The kinetics of the austenite $\rightarrow$ martensite transformation could be followed with a good resolution. Parallely, the evolution of lattice parameters of austenite and martensite were determined and used to follow the generation of stresses during quenching.

\section{Experimental}

In situ X-ray diffraction experiments were performed at the European Synchrotron Radiation Facility (ESRF) in Grenoble, France, on beam line ID11. The experiments were executed with a heating device (ETMT, Instron) allowing a controlled heating of samples with different cross section of $1 \mathrm{~mm}, 1.5 \mathrm{~mm}$ and $2 \mathrm{~mm}$ with a length of $40 \mathrm{~mm}$ by resistivity. An AISI52100 (100Cr6) steel with following chemical composition Fe-0.95C-1.45Cr-0.44Mn-0.21 Si-0.11Ni-0.10Cu- 
$0.05 \mathrm{Mo}-0.01 \mathrm{P}-0.005 \mathrm{~S}-0.004 \mathrm{Al}$ Mass- $\%$ was used. The initial microstructure of the steel exhibits a ferritic matrix with globular carbides.

Following heat treatment cycle was used: heating with a rate of $5 \mathrm{~K} / \mathrm{s}$ up to different austenitizing temperature (TA) between $800{ }^{\circ} \mathrm{C}$ and $940{ }^{\circ} \mathrm{C}$ followed by a soaking time of $15 \mathrm{~min}$ and quenching to room temperature (RT). The clamping tools huge mass allowed quenching of samples without use of any other quenching media. The different samples cross section led to slightly varying cooling rates with $t 8 / 5$ times between $2.18 \mathrm{~s}$ for $1 \mathrm{~mm}$ cross section and $2.82 \mathrm{~s}$ for 2 $\mathrm{mm}$. Constant argon purging was used to avoid oxidation of the surface. The temperature was controlled by using a type $\mathrm{K}$ thermocouple welded on the surface of the samples.

During the entire heat treatment cycle, diffraction frames were recorded in transmission mode with a FRELON camera [11] using an exposition time of 0.7 seconds for each frame during quenching. The beam energy was $71 \mathrm{keV}$ and the primary beam size was set at maximum (about $100 \mu \mathrm{m}$ high and $300 \mu \mathrm{m}$ width) in order to get the best statistical conditions in terms of diffracting domains. A standard material (LaB6 powder) was measured to describe the instrumental contribution on the diffraction patterns. The recorded frames were integrated with the program Fit2d developed at ESRF after background correction for further analysis [12]. The analysis of diffraction patterns after integration has been performed with the Rietveld refinement software TOPASC from Bruker AXS. X-ray analysis of a fine polycrystalline material will result in a convolution of all microstructural features [13]. Different phases are present here and each phase may contribute in different way to the diffraction pattern according to local textures, anisotropic size and strain broadening as well as stacking faults. Microstrain and size broadening was introduced to model the peak widths. The refinement parameters obtained were fixed and used for the analysis of the temperature dependent diffraction patterns obtained during quenching.

\section{Results and discussion}

The 2d-diffraction frames recorded at different temperatures during quenching of a sample austenitized at $915{ }^{\circ} \mathrm{C}$ is presented in Fig.1a. It can be observed that at high temperature, the diffraction rings are not completely homogeneous but spots are present. This is due to the grain coarsening leading to a decreasing sampling statistic at high temperature. During quenching, no significant changes of the diffraction rings can be observed until Ms is reached $\left(170{ }^{\circ} \mathrm{C}\right)$. Then, diffraction rings belonging to martensite appear, and the diffraction rings of austenite become more and more close due to a plastic deformation and related increasing number of crystallites.
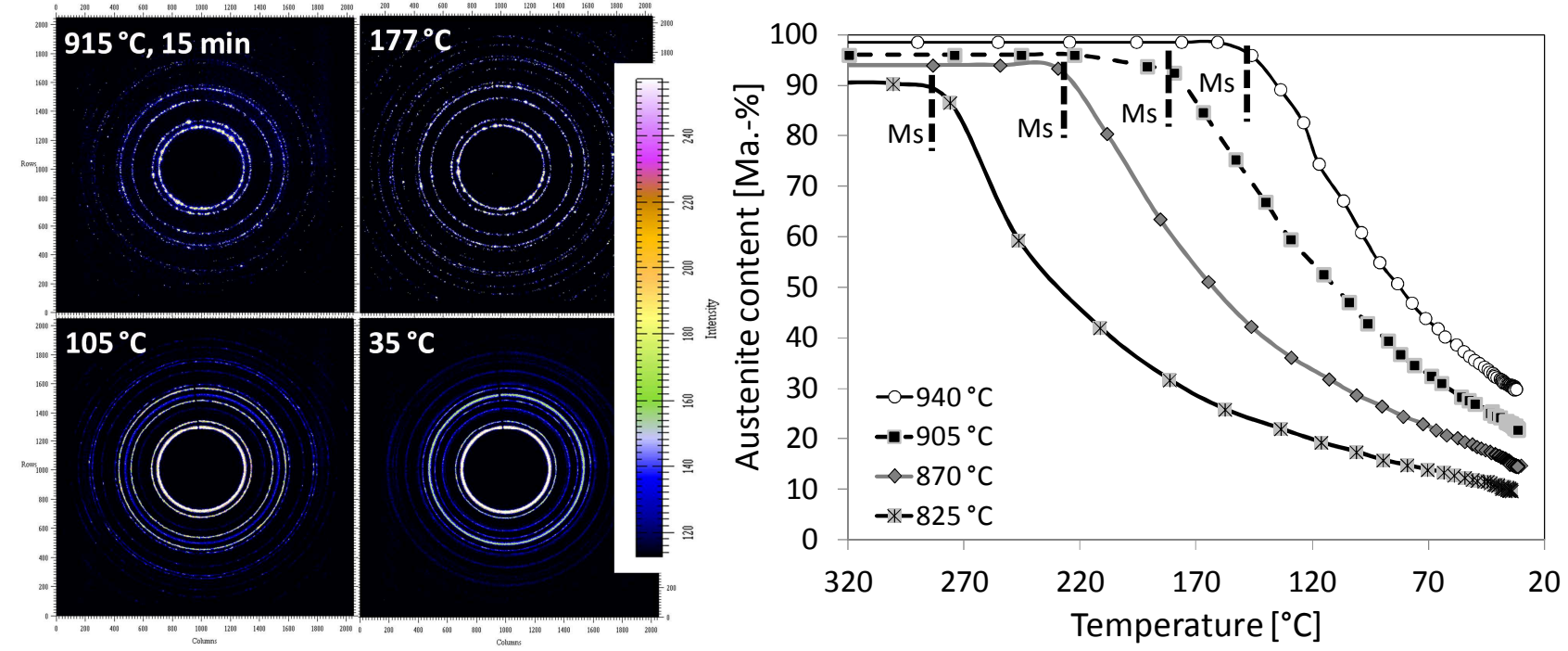

Fig. 1: a) 2d-diffraction frames taken at different temperatures during quenching of a sample after austenitizing at $915^{\circ} \mathrm{C}$; b) evolution of austenite content during quenching of 4 samples after austenitizing at different temperatures between 825 and $940{ }^{\circ} \mathrm{C}$

After integration of the 2d-diffraction frames and analysis of the standard intensity-vs 2Theta diffractograms by the Rietveld method, the evolution of phase content could be precisely described. 
Fig1b shows the evolution of austenite content during quenching for different initial austenitizing temperatures. At austenitizing temperature, undissolved carbides $\left(\mathrm{Fe}_{3} \mathrm{C}\right)$ still remain in the microstructure. This explains the fact that the austenite amount is not at $100 \%$, and that the initial austenite content is increasing with increasing austenitizing temperature. The kinetics of the martensitic transformation could be well followed. At the end of the quenching, the amount of retained austenite present in the microstructure is 9, 14, 21 and 30 Mass.- $\%$ for the austenitizing temperatures $825,870,905$ and $940{ }^{\circ} \mathrm{C}$ respectively.

From the Rietveld refinements, the lattice parameters of austenite could be extracted and are plotted as a function of the temperature during quenching of the considered 4 samples austenitized at different temperatures between 825 and $940^{\circ} \mathrm{C}$. With increasing austenitizing temperature, the lattice parameters are shifted to higher values due to increasing carbon content in solution, as expected. The decrease with decreasing temperature is linear and almost parallel for all experiments until Ms is reached. During further cooling, it can be observed that a change of slope takes place with a stronger decrease towards room temperature. From the measured lattice parameters evolutions, the carbon content in solid solution in austenite can be estimated by using, the equation of Onink (Eq. 1) [14]. In that equation, the effect of the carbon content (fc in at.-\%) on the thermal expansion coefficient is taken into account. The temperature $\mathrm{T}$ is in Kelvin.

$a_{\gamma}\left(f_{C}, T\right)=\left(0.363067+0.000783 \times f_{C}\right) \times\left(1+\left(24.92-0.51 \times f_{C}\right) \times 10^{-6}[T-1000]\right)$.

After conversion to Mass.- $\%$, the determined evolution of carbon content in solid solution in austenite during quenching of the 4 samples already presented in Fig $2 \mathrm{a}$ are shown in Fig $2 \mathrm{~b}$. For all samples a similar behavior can be observed: the determined carbon content in solution is almost constant at values between 0.6 and 1.0 Mass- $\% \mathrm{C}$ according to the austenitizing temperature until $\mathrm{Ms}$ is reached. At slight undercooling below Ms, the calculated carbon content in solution exhibits a continuous decrease until room temperature is reached. The total decrease is different for the four austenitizing temperatures. As the cooling rate is high and Ms is very low (in particular for austenitizing above $900{ }^{\circ} \mathrm{C}$ ), it appears improbable that such strong carbon loss due to selftempering can occur in austenite during quenching. Therefore, the only plausible explanation for the strong decreasing lattice parameter below $\mathrm{Ms}$ is the generation of strong hydrostatic residual stresses in austenite.
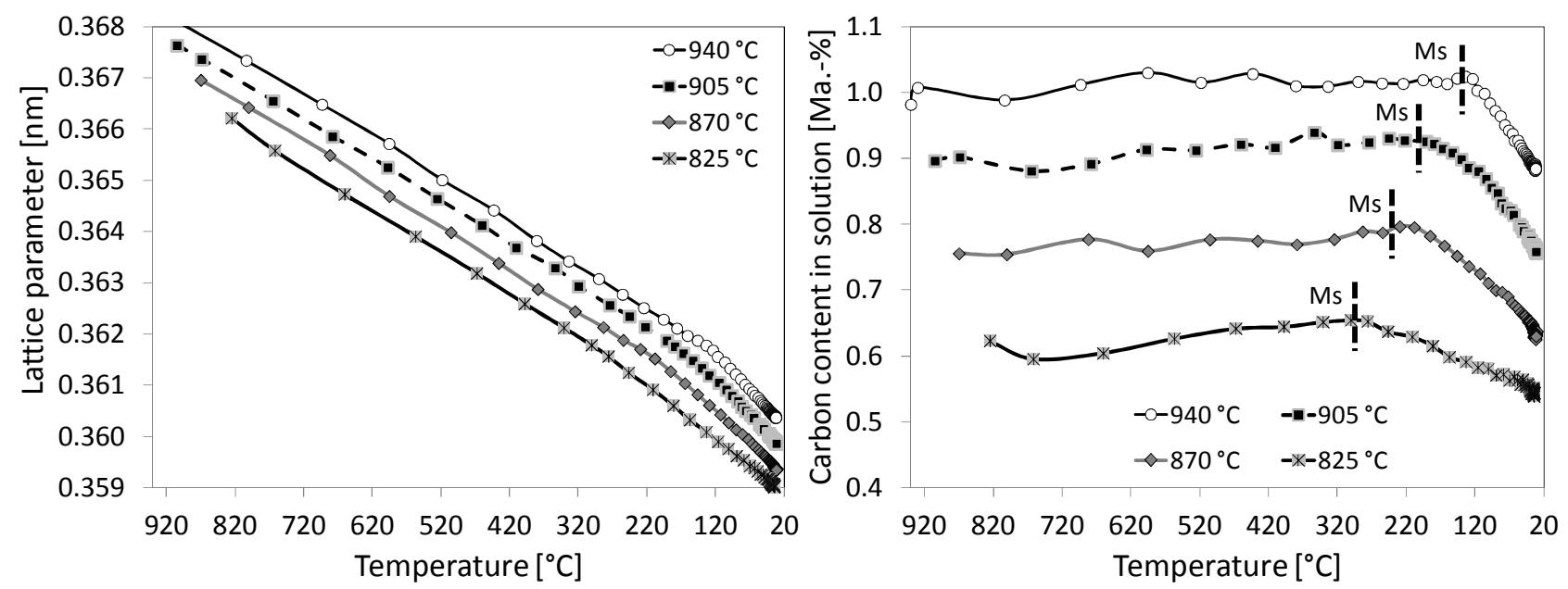

Fig. 2: a) evolution of austenite lattice parameter during quenching after austenitizing at different temperatures between 825 and $940^{\circ} \mathrm{C}$; b) corresponding evolution of carbon content in solution calculated on the base of the measured lattice parameters for the different austenitizing temperatures (the decreasing carbon content below Ms is not a real effect but is attributed to the generation of hydrostatic stresses)

Few studies can be found in the literature about residual stresses in retained austenite during or after quenching of steel. Several authors describe the residual stress state within retained austenite existing at room temperature as a hydrostatic residual stress state under high compressive stresses $[7,8,9]$. The reason for this would be the very large volume expansion associated with the 
martensititic transformation ( $>3 \%$ ). Of course, due to shear-processes during the martensitic transformation, local residual stresses will not be purely hydrostatic in a single austenite region. However, X-ray diffraction techniques give average information of thousands (millions) of "grains" which in general are randomly oriented, and therefore the average information contained in the measured area might be predominantly hydrostatic.

In the literature, two different sets of data concerning the evolution of austenite lattice parameter at room temperature as a function of the carbon content can be found: the one based on room temperature measurements after quenching (retained austenite), and the one based on high temperature measurements of austenite with thermal expansion correction. From the literature data, equations were developed based on the room temperature measurements $\left(\mathrm{a}^{\mathrm{RT}}=0.3556+\right.$ $0.00443 \times \% \mathrm{C})$ and on the high temperature measurements $\left(\mathrm{a}^{\text {stress-free }}=0.3573+0.00327 \times \% \mathrm{C}\right.$ ) with $\% \mathrm{C}$ in Mass.-\% [7]. It can be assumed that the main difference between the high temperature data and the measurements of retained austenite at room temperature is the possible generation of hydrostatic residual stresses as a consequence of the large transformation strain and the different thermal expansion coefficients of austenite and martensite.

Both equations from the literature can be used to quantify the possible existing hydrostatic residual stress state in retained austenite depending on the carbon content in solution. If it is assumed that the high temperature lattice parameters describe the stress-free state while the equation based on room temperature measurements represents the retained austenite under stress after quenching, Eq. 2 can be used to calculate the corresponding strain $\left(\varepsilon^{\mathrm{RA}}\right)$ in retained austenite.

$$
\varepsilon^{R A}=\frac{\Delta a}{a^{\text {stress-free }}}=\frac{a^{R T}-a^{\text {stress-free }}}{a^{\text {stress-free }}} .
$$

Using Eq. 2, the resulting hydrostatic residual stresses at room temperature can be calculated by Eq. 3 with $\mathrm{E}^{\mathrm{RA}}=207 \mathrm{GPa}$ and $v^{\mathrm{RA}}=0.28$.

$$
\sigma^{R A}=\varepsilon^{R A} \times \frac{E^{R A}}{\left(1-2 v^{R A}\right)} .
$$

The evolution of the literature data of the stress-free lattice parameter of retained austenite $\left(a^{\text {stress-free }}\right)$ and of the lattice parameter of retained austenite after quenching $\left(a^{\mathrm{RT}}\right)$ depending on the carbon content in solution is presented in Fig 3a. From these theoretical evolutions, it can be observed that at values close to 1.5 Mass- $\%$ C both lattice parameters are almost similar and with decreasing carbon content, the gap is increasing continuously. From these data, and together with Eq. 2 and 3, resulting theoretical hydrostatic residual stresses were calculated and plotted in Fig. $3 \mathrm{~b}$. At 1.5 Mass- $\%$ C, almost no residual stresses were determined as $\Delta \mathrm{a}$ is very small. With decreasing carbon content in solution, the determined values go to always increasing compressive residual stresses. For carbon-free steel $(0 \% \mathrm{C})$ a theoretical value of $-2300 \mathrm{MPa}$ is obtained. Of course it is thermodynamically almost impossible to have retained austenite in low alloy steels without carbon. However, it appears from these calculations that according to the data of the literature, retained austenite might be under very large hydrostatic compressive residual stresses after quenching.

The experimental data collected at RT within the frame of the present study were then used to calculate hydrostatic residual stresses in retained austenite based on Eq. 2 and 3 and using the stress-free lattice parameter from the literature, and plotted in Fig. 3b. It can be observed that the experimental values are close to the theoretical line obtained from the literature data for carbon content in solution above 0.75 Mass- $\% \mathrm{C}$, with values between $-750 \mathrm{MPa}$ to $-300 \mathrm{MPa}$, but with a shift. Discrepancies between the experimental values (triangles) and the theoretical evolution (black line) in this range of carbon content $(>0.75 \% \mathrm{C})$ can either result from an influence of alloying elements on lattice parameters, that can lead to slight variations of the stress-free references, or from a systematic shift in the lattice parameter determination due to the experimental setup.

When the carbon content in solution decreases below 0.75 Mass- $\%$, a continuous deviation of the experimental values from the theoretical line can be observed. This deviation might be a consequence of carbon enrichment of the austenite due to the fact that with decreasing $\% \mathrm{C}, \mathrm{Ms}$ 
increases and therefore carbon diffusion is accelerated. Similar behaviors were already reported in low to medium carbon steels $[1,15,16]$.

As already observed previously, during the present experiments with $100 \mathrm{Cr} 6$ samples, the measured lattice parameters of retained austenite during quenching show a non-linear change of the slope (continuous decrease) below Ms (Fig. 2b). This resulted in continuously decreasing calculated values of carbon contents in solution in austenite. However, the change of slope of austenite lattice parameter at temperatures below Ms can be attributed to the generation of large compressive residual stresses. Based on the present in situ experiments, it is possible to estimate the development of hydrostatic stresses in austenite during quenching. In order to obtain the temperature-dependent stress-free lattice parameter, a linear extrapolation of the austenite lattice parameter measured in the present experiments at temperatures above Ms down to RT was used (evolution presented in Fig 2a). For the further evaluations, only the experiments with initial carbon content in solution above 0.75 Mass.- $\% \mathrm{C}$ will be considered, as the effect of possible carbon enrichment for lower carbon contents in solution cannot be taken into account at this point of the investigations. The stress calculations were performed by using Eq. 2 and 3 at each temperature during cooling, taking into account the temperature-dependent changes of elastic properties as given by [17].
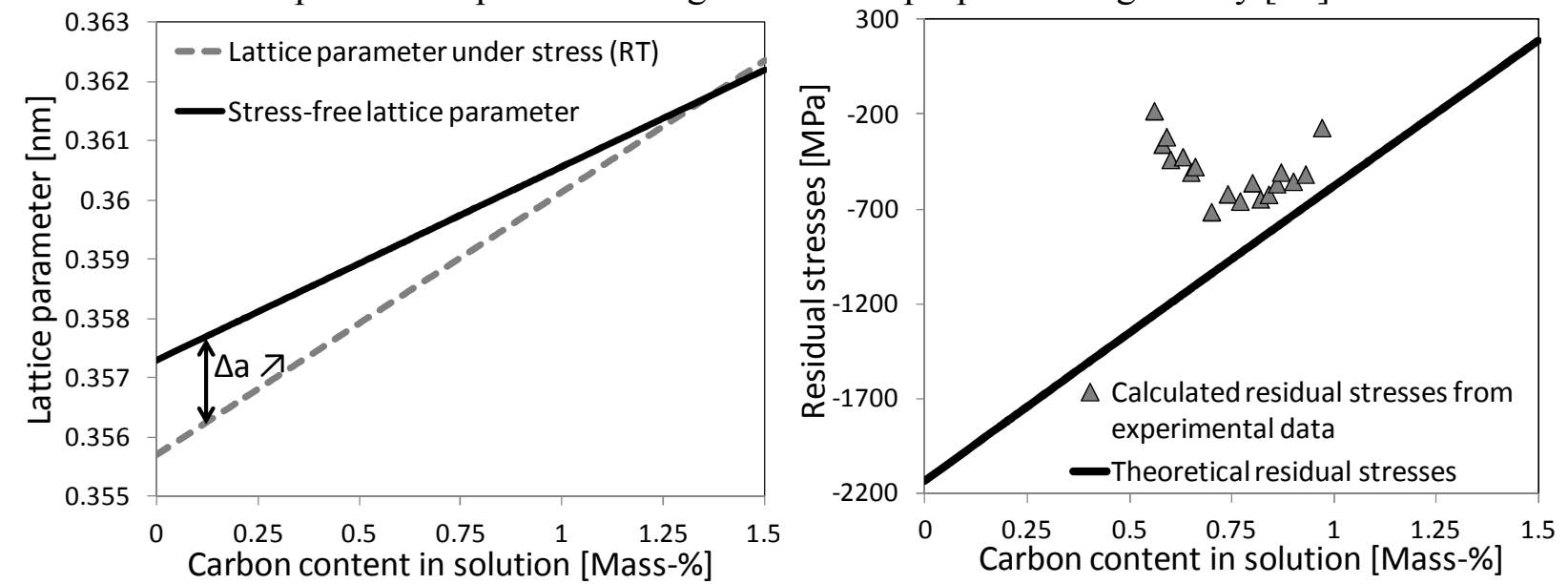

Fig. 3: a) Evolution of the stress-free ( $\left.a^{\text {stress-free }}\right)$ and the RT austenite lattice parameters $\left(a^{R T}\right)$ as a function of the carbon content in solution (data from the literature); b) hydrostatic residual stresses resulting from both evolution in (a) and values calculated from lattice parameters measured in the present in situ experiments using stress-free lattice parameters from the literature
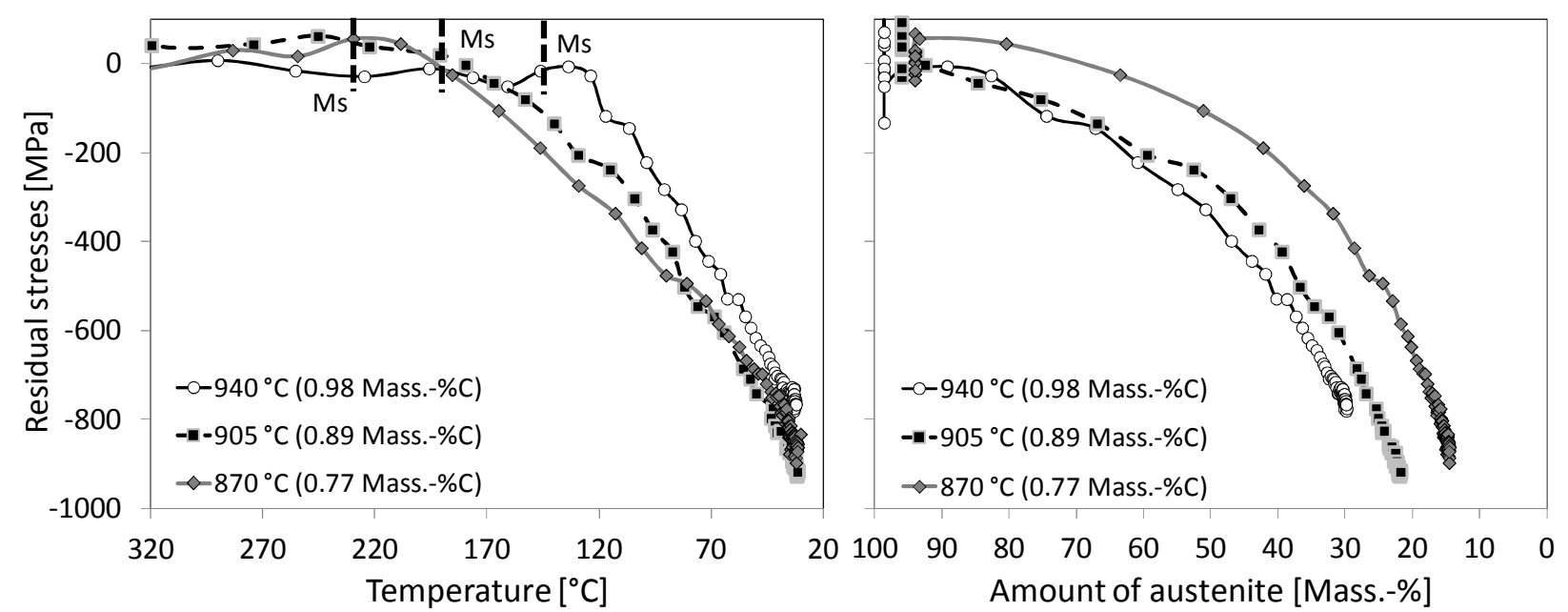

Fig.4: Development of hydrostatic stresses in austenite during quenching of 100Cr6 samples with different austenitizing temperatures as a function of the temperature (a) and of the amount of retained austenite (b)

The evolutions of hydrostatic compressive stresses in austenite during cooling of several experiments with C-contents above 0.75 Mass.- $\%$ with direct cooling are presented in Fig. $4 \mathrm{a}$. It can be observed that the increase of compressive stresses starts after a slight undercooling below Ms, 
where the compressive stresses increase continuously with decreasing temperature until RT is reached. Towards room temperature it can be observed that small cooling steps lead to a large increase of the compressive stresses. The residual stress values that are reached at RT after cooling are in the range of -920 for austenitizing at $905^{\circ} \mathrm{C}$ to $-770 \mathrm{MPa}$ after austenitizing at $940{ }^{\circ} \mathrm{C}$.

Discrepancies between the experimental values in Fig $3 \mathrm{~b}$ (triangles) and end values at RT in Fig 4 can be explained by the already mentioned influence of alloying elements and shifts due to the experimental setup, as in Fig 3, the stress-free lattice parameters were obtained by using the literature data while in Fig. 4, the measured high temperature lattice parameters were extrapolated to RT and used as the stress-free values. Therefore, the values obtained in Fig 4 are considered to be more reliable. Furthermore, the residual stress values at RT in Fig 4 are very close to the theoretical evolution obtained from the literature data in the considered range of carbon content (Fig $3 \mathrm{~b}$ ).

The generation of large hydrostatic compressive stresses in austenite during the martensitic transformation is assumed to be related to the volume change due to martensitic transformation. Therefore, the plot of the evolution of compressive stresses in austenite as a function of the amount of retained austenite (Fig 4b) can give additional information. Here, it can be noticed that for all experiments, only a slow increase of residual stresses occurs for less than 40 Mass- $\%$ transformed austenite. For increasing amounts of transformed austenite, the increase of compressive stresses follows an almost exponential behavior. The present measurements give average information from a volume of $300 \times 300 \times 1500 \mu^{3}$. When only a small amount of austenite transformed into martensite, the austenite regions that are unaffected by the transformation strain are much larger than the affected region. The average signal change that is measured is therefore moderate. When the amount of austenite that was affected by the formed martensitic plates is increasing, the recorded peak shifts (due to stresses) is getting larger. This effect is superimposed to the proper effect of increasing strains in austenite when an increasing part of the surrounding regions transforms to martensite. Due to both effects, the detected increasing compressive stress exhibits a strong dependence on the amount of transformed austenite.

In order to verify if large compressive hydrostatic residual stresses are present in retained austenite after quenching, measurements of complete residual stress tensors were performed at several disc-shaped samples from $100 \mathrm{Cr} 6$ steel, austenitized at different temperatures. The measurements were performed with a laboratory diffractometer with $\mathrm{Cr}-\mathrm{k} \alpha$ radiation at the $\{220\}$ lattice planes of austenite, using respectively $35 \psi$ angles between $-67.5^{\circ}$ and $67.5^{\circ}$ for $3 \varphi$ angles $\left(0,45,90^{\circ}\right)$. The stress free lattice spacing of austenite $\mathrm{d}_{0}$ was calculated by using the literature data as presented in Fig 3a. Two residual stress tensors were determined:

Austenitizing $940{ }^{\circ} \mathrm{C}:\left(\begin{array}{ccc}-740 & 4 & 7 \\ 4 & -715 & -4 \\ 7 & -4 & -725\end{array}\right) \quad$ Austenitizing $880{ }^{\circ} \mathrm{C}:\left(\begin{array}{ccc}-1061 & 16 & 11 \\ 16 & -1065 & -4 \\ 7 & -4 & -1040\end{array}\right)$

The carbon content in solution above Ms was about 0.98 Mass- $\% \mathrm{C}$ for austenitizing at $940{ }^{\circ} \mathrm{C}$, and 0.84 Mass- $\% \mathrm{C}$ for austenitizing at $880{ }^{\circ} \mathrm{C}$. It can be observed that the determined residual stress tensors are all almost purely hydrostatic and that the stress values are comparable to the experimental values obtained during in situ experiments (Fig 4) and to the theoretical calculations as given in Fig 3b. In general, for X-ray diffraction measurements with laboratory equipment, a plane stress state is assumed as the penetration of the X-ray beam into the sample is very shallow (3 to $5 \mu \mathrm{m}$ for $\mathrm{Cr}-\mathrm{k} \alpha$ radiation in steel) [18]. However, when a multiphase material with a periodically distributed second phase is measured, the macroscopic stress component normal to the surface is zero but $2^{\text {nd }}$ kind residual stresses in the present phases can be non-zero with opposite signs [19]. This is the case when the period of distribution of the second phase is of the same order of magnitude as the penetration depth of the radiation [19]. As the values measured by the X-ray diffraction with $\mathrm{Cr}-\mathrm{k} \alpha$ radiation are in the same range as the values obtained from the in situ experiments and from the calculations based on literature data, it can be concluded that in hardened steel, the retained austenite (and therefore the martensite) exhibits a triaxial, mostly hydrostatic residual stress state, even when the penetration depth of the radiation is below $5 \mu \mathrm{m}$. 


\section{Summary}

In situ XRD experiments were performed during quenching of a ball bearing steel grade AISI 52100 (100Cr6) after austenitizing at different temperatures leading to varying amount of carbon in solid solution and therefore to changes in the amount of retained austenite. By investigating the evolution of austenite lattice parameter during the quenching process, it could be observed that a linear decrease takes place until Ms is reached and during further cooling a change of slope occurs. From the lattice parameters, constant values of carbon content in solid solution according to the austenitizing temperature were determined until Ms is reached and then a continuous decrease towards room temperature could be observed. However, this decrease was considered to be not-real, but rather be attributed to the generation of almost hydrostatic compressive residual stresses in the austenitic phase. This assumption is supported by calculated hydrostatic residual stresses based on data from the literature for high-temperature austenite and measurements of retained austenite at room temperature. With the present in situ experiments, the evolution of hydrostatic stresses in austenite could be followed during the whole quenching process and showed the generation of large compressive residual stresses up to $-920 \mathrm{MPa}$. Comparable values were determined by laboratory XRD residual stress tensor measurements, showing that even within the small penetration depth of $\mathrm{Cr}-\mathrm{k} \alpha$ radiation, non-zero $\sigma_{33}$ component is present in retained austenite. In future works, the generation of stresses in martensite and the effect of $\mathrm{C}$-enrichment in austenite will be addressed.

\section{Acknowledgement}

The authors gratefully acknowledge the Deutsche Forschungsgemeinschaft (DFG) for financial support of project C01 in the Transregional Collaborative Research Centre 136 „Process Signature” as well as Loredana Erra and the ESRF for support and provision of synchrotron radiation facilities.

\section{References}

[1] G.B. Olson, W.S. Owen: Martensite, first ed., ASM International, 1992.

[2] Z. Nishiyama, M.E. Fine, M. Meshii, C.M. Wayman: Martensitic Transformation, Academic Press, New York, 1978.

[3] G.V. Kurdjumov, Metall. Mater. Trans. A, 7 (1976) 999-1011.

[4] Metals Handbook, Vol. 1: Properties and Selection: Irons, Steels, and High-Performance Alloys, tenth ed., ASM International 1990

[5] C. Acht, B. Clausen, F. Hoffmann, H.W. Zoch in: H.W. Zoch, Th. Lübben, (Eds.) Proc. 1st Int. Conf. on Distortion Engineering, 14-16.09.2005, Bremen, Germany, 251-258.

[6] F. Frerichs, Th. Lübben, F. Hoffmann, H.-W. Zoch, Steel Res. Int, 78-7 (2007), 558-563.

[7] L. Cheng, A. Böttger, T. H. deKeijser, E.J. Mittemeijer, Scripta Metall Mater, 24 (1990) 509514.

[8] Y. Liu, Z. L. Xie, H. Hiinninen, J. Van Humbeeck, J. Pietiktiinen, J. Phys. IV, 5-1 (1995) 179184.

[9] K. Y. Golovchiner, Physics of Metals and Metallography, 37-2 (1974) 126-130.

[10] J. Epp, H. Surm, O. Kessler, T. Hirsch, Metal. Mater, Trans A, 38-10 (2007) 2371-2378.

[11] J.-C. Labiche, O. Mathon, S. Pascarelli, M. A. Newton, G. Guilera Ferre, C. Curfs, G. Vaughan, A. Homs, chemistry, and catalysis, Review Sci. Inst, 78 (2007)

[12] A. Hammersley, S.O. Svensson, A. Thompson, Nucl. Instrum. Methods A, 346 (1994) 312.

[13] B.E. Warren: X-Ray Diffraction, Addison-Weseley, Reading, Mass., 1969.

[14] M. Onink, C. M. Brakman, F. D. Tichelaar, E. J. Mittemeijer, S. van der Zwaaag, Acta Metall et Mat, 29 (1993) 1011-1016.

[15] D.H. Sherman, S.M. Cross, S. Kim, F. Grandjean, G.J. Long, M.K. Miller, Metall. Mater. Trans. A, 38 (2007) 1698-1711.

[16] J. Epp, T. Hirsch, C. Curfs, Metallurgical and Materials Transaction A, 43 (2012) 2210 - 2217.

[17] F. Richter: Verlag Stahleisen GmbH, Düsseldorf, 1983

[18] V. Hauk, Structural and residual stress analysis by nondestructive methods, Elsevier Science, Amsterdam, 1997

[19] H. Behnken, Mikrospannungen in vielkristallinen und heterogenen Werkstoffen, Shaker Verlag, 2003 